\title{
The Inviscid Limit Behavior for Smooth Solutions of the Boussinesq System
}

\author{
Junlei Zhu \\ College of Mathematics, Physics and Information Engineering, Jiaxing University, Zhejiang 314001, China \\ Correspondence should be addressed to Junlei Zhu; zhujl-001@163.com
}

Received 13 January 2013; Accepted 30 March 2013

Academic Editor: Jesús I. Díaz

Copyright (C) 2013 Junlei Zhu. This is an open access article distributed under the Creative Commons Attribution License, which permits unrestricted use, distribution, and reproduction in any medium, provided the original work is properly cited.

The inviscid limit problem for the smooth solutions of the Boussinesq system is studied in this paper. We prove the $H^{s}$ convergence result of this system as the diffusion and the viscosity coefficients vanish with the initial data belonging to $H^{s}$. Moreover, the $H^{s}$ convergence rate is given if we allow more regularity on the initial data.

\section{Introduction and the Main Results}

The two-dimensional Boussinesq system for the homogeneous incompressible fluids with diffusion and viscosity is given by

$$
\begin{gathered}
\mathbf{u}_{t}+(\mathbf{u} \cdot \nabla) \mathbf{u}+\nabla p=v \Delta \mathbf{u}+\theta \mathbf{e}_{2}, \\
\theta_{t}+(\mathbf{u} \cdot \nabla) \theta=\kappa \Delta \theta, \\
\nabla \cdot \mathbf{u}=0, \\
\left.u\right|_{t=0}=u_{0},\left.\quad \theta\right|_{t=0}=\theta_{0},
\end{gathered}
$$

where the space variable $\mathbf{x}=\left(x_{1}, x_{2}\right)$ is in $\mathbb{R}^{2}$, $\mathbf{u}=$ $\left(u^{1}(t, \mathbf{x}), u^{2}(t, \mathbf{x})\right)$ is the velocity, $p=p(t, \mathbf{x})$ denotes the scalar pressure and $\theta=\theta(t, \mathbf{x})$ the scalar temperature, $\mathbf{e}_{2}=(0,1)$, and $\kappa>0$ and $\nu>0$ denote, respectively, the molecular diffusion and the viscosity. Such Boussinesq systems are simple models widely used in the modeling of oceanic and atmospheric motions, and these models also appear in many other physical problems; see $[1,2]$ for more discussions. It is also interesting to consider the system (1) without diffusion and viscosity namely (for the sake of convenience for our limit argument, we use different notation),

$$
\begin{gathered}
\mathbf{v}_{t}+(\mathbf{v} \cdot \nabla) \mathbf{v}+\nabla \pi=\rho \mathbf{e}_{2}, \\
\rho_{t}+(\mathbf{v} \cdot \nabla) \rho=0,
\end{gathered}
$$

$$
\begin{gathered}
\nabla \cdot \mathbf{v}=0, \\
\left.\mathbf{v}\right|_{t=0}=\mathbf{v}_{0},\left.\quad \rho\right|_{t=0}=\rho_{0} .
\end{gathered}
$$

Moreover, it is known that the two-dimensional viscous (resp., inviscid) Boussinesq equations are closely related to the three-dimensional axisymmetric Navier-Stokes equations (resp., Euler equations) with swirl. Therefore, the Boussinesq systems, especially in two-dimensional case, have been widely studied by many researchers, and we refer, for instance, to [39] and the references therein.

It is well known that the system (1) has a unique global in time regularity solution. Moreover, Hou and $\mathrm{Li}$ in [9] obtained the global existence of smooth solution for (1) even with the zero diffusivity case (i.e., $v>0$ and $\kappa=0$ ). Meanwhile, Chae in [5] also proved global regularity for the 2D Boussinesq system (1) both with the zero diffusivity case $(\nu>0$ and $\kappa=0)$ and the zero viscosity case $(\nu=0$ and $\kappa>0$ ). However, for the case $v=0$ and $\kappa=0$, it is only known that smooth solution exists locally in time (see, e.g., [4]), and it is not known whether such smooth solutions can develop singularities in finite time. In fact, as well as the famous blow-up problem for the Navier-Stokes equations or Euler equations, the regularity or singularity question for the locally smooth solution of the system (2) appears also as an outstanding open problem in the mathematical fluid mechanics; see [10]. 
In this paper, we are interested in studying the limit behavior of the smooth solution for $(1)$ as $(\nu, \kappa) \rightarrow 0$; that is, we study the vanishing viscosity limit of solutions of (1). This type limit problem appears not only in the community of applied mathematics, but also in physical reality. A good example of this problem is the vanishing viscosity limit of solutions of the Navier-Stokes equations which appears as a singular limit especially in bounded domains due to the boundary layers effect, and we refer to [11-17] and the references therein. Most of the previous convergence results require some loss of derivatives; namely, if the initial data lies in the space $H^{s}$, usually one can obtain the convergence results in $H^{s^{\prime}}$ with $s^{\prime}<s$. In this literature, Masmoudi in [15] obtained the inviscid limit results for the Navier-Stokes equations without loss of derivatives. Inspired by [15], in this paper, we obtain the $H^{s}$ convergence of the solution with the initial data belonging to the same space.

Now we state our main results of the paper.

Theorem 1. Let $s>3,\left(\mathbf{u}_{0}^{\nu, \kappa}, \theta_{0}^{v, \kappa}\right) \in \mathbf{H}^{s}\left(\mathbb{R}^{2} ; \mathbb{R}^{2}\right) \times H^{s}\left(\mathbb{R}^{2}\right)$, and $\left(\mathbf{v}_{0}, \rho_{0}\right) \in \mathbf{H}^{s}\left(\mathbb{R}^{2} ; \mathbb{R}^{2}\right) \times H^{s}\left(\mathbb{R}^{2}\right)$ satisfying $\nabla \cdot \mathbf{u}_{0}^{\nu, \kappa}=0$, $\nabla \cdot \mathbf{v}_{0}=0$, and

$$
\mathbf{u}_{0}^{v, \mathcal{K}} \longrightarrow \mathbf{v}_{0}, \quad \theta_{0}^{\gamma, \mathcal{K}} \longrightarrow \rho_{0} \quad \text { in } H^{s},
$$

as $(\nu, \kappa) \rightarrow 0$. Assume that $\left(\mathbf{u}^{\nu, \kappa}, \theta^{\nu, \kappa}\right) \in C\left(\mathbb{R}^{+} ; \mathbf{H}^{s} \times H^{s}\right)$ is the classical solution of (1) with initial data $\left(\mathbf{u}_{0}^{\nu, \kappa}, \theta_{0}^{\gamma, \kappa}\right)$, and $(\mathbf{v}, \rho) \in C\left(\left[0, T^{*}\right) ; \mathbf{H}^{s} \times H^{s}\right)$ is the classical solution of the inviscid system (2) with initial data $\left(\mathbf{v}_{0}, \rho_{0}\right)$; here; $T^{*}$ is the maximal existence time of the solution $(\mathbf{v}, \rho)$. Then, for any $T_{0} \in\left(0, T^{*}\right)$ and for any $t \in\left[0, T_{0}\right]$, one has

(1) (convergence rate in the $H^{s-2}$ norm)

$$
\begin{aligned}
& \left\|\mathbf{u}^{\nu, \mathcal{K}}(t)-\mathbf{v}(t)\right\|_{\mathbf{H}^{s-2}}+\left\|\theta^{\nu, \mathcal{\kappa}}(t)-\rho(t)\right\|_{H^{s-2}} \\
& \quad \leq C_{1}\left(\nu+\kappa+\left\|\mathbf{u}_{0}^{\nu, \kappa}-\mathbf{v}_{0}\right\|_{\mathbf{H}^{s-2}}+\left\|\theta_{0}^{\nu, \kappa}-\rho_{0}\right\|_{H^{s-2}}\right),
\end{aligned}
$$

(2) (convergence rate in the $H^{s_{1}}$ norm with $s-2<s_{1} \leq$ $s-1)$

$$
\begin{gathered}
\left\|\mathbf{u}^{\nu, \kappa}(t)-\mathbf{v}(t)\right\|_{\mathbf{H}^{s_{1}}}+\left\|\theta^{\nu, \kappa}(t)-\rho(t)\right\|_{H^{s_{1}}} \\
\leq C_{2}\left(\nu^{\left(s-s_{1}\right) / 2}+\kappa^{\left(s-s_{1}\right) / 2}+\left\|\mathbf{u}_{0}^{\nu, \kappa}-\mathbf{v}_{0}\right\|_{\mathbf{H}^{s_{1}}}\right. \\
\left.+\left\|\theta_{0}^{\nu, \kappa}-\rho_{0}\right\|_{H^{s_{1}}}\right),
\end{gathered}
$$

(3) (convergence rate in the $H^{s_{2}}$ norm with $s-1<s_{2}<s$ )

$$
\begin{gathered}
\left\|\mathbf{u}^{\nu, \kappa}(t)-\mathbf{v}(t)\right\|_{\mathbf{H}^{s_{2}}}+\left\|\theta^{\nu, \kappa}(t)-\rho(t)\right\|_{H^{s_{2}}} \\
\leq C_{3}\left(\nu^{\left(s-s_{2}\right) / 2}+\kappa^{\left(s-s_{2}\right) / 2}+\left\|\mathbf{u}_{0}^{\nu, \kappa}-\mathbf{v}_{0}\right\|_{\mathbf{H}^{s-1}}^{s-s_{2}}\right. \\
\left.+\left\|\theta_{0}^{\nu, \mathcal{\kappa}}-\rho_{0}\right\|_{H^{s-1}}^{s-s_{2}}\right)
\end{gathered}
$$

(4) (convergence in the $H^{s}$ norm)

$$
\begin{aligned}
\left\|\mathbf{u}^{\nu, \kappa}-\mathbf{v}\right\|_{C\left(\left[0, T_{0}\right] ; \mathbf{H}^{s}\right)} & \\
& +\left\|\theta^{\nu, \kappa}-\rho\right\|_{C\left(\left[0, T_{0}\right] ; H^{s}\right)} \longrightarrow 0 \text { as }(\nu, \kappa) \longrightarrow 0,
\end{aligned}
$$

where the constants $C_{i}(i=1,2,3)$ in (4)-(6) are dependent of $T_{0}$ and the $H^{s}$ norm of the initial data $\left(\mathbf{v}_{0}, \rho_{0}\right)$, but independent of the parameters $\nu$ and $\kappa$.

Of course, we can generalize the previous results to arbitrary spatial dimension case with $s>3$ replaced by $s>$ $(n / 2)+2$. The important part of Theorem 1 is the convergence result (7). This result tells us that the $H^{s}$ convergence can be maintained by the solution at its arbitrary existence time. We emphasize that $T^{*}$ is not assumed to be small; indeed, the standard energy estimate yields that the classical solution $(\mathbf{v}, \rho)$ blows up at time $T^{*}$ if and only if $\|\mathbf{v}(t)\|_{\mathbf{H}^{s}}+\|\rho(t)\|_{H^{s}} \rightarrow$ $\infty$ as $t \uparrow T^{*}$. Note that the rate of $H^{s}$ convergence depends on how we regularize our initial data; see (75) in the next section. Moreover, if one allows more regularity on the initial data $\left(\mathbf{v}_{0}, \rho_{0}\right)$, then we can obtain the following $H^{s}$ convergence rate.

Theorem 2. Suppose that the same assumptions as Theorem 1 hold. Moreover, one assumes that $\left(\mathbf{v}_{0}, \rho_{0}\right) \in \mathbf{H}^{s+\delta} \times H^{s+\delta}$ with $\delta \in(0,2]$. Then, for any $t \in\left[0, T_{0}\right]$, there hold

$$
\begin{aligned}
& \left\|\mathbf{u}^{\gamma, \kappa}(t)-\mathbf{v}(t)\right\|_{\mathbf{H}^{s}}+\left\|\theta^{\gamma, \kappa}(t)-\rho(t)\right\|_{H^{s}} \\
& \leq C_{4}\left(v^{\delta / 2}+\kappa^{\delta / 2}+\left\|\mathbf{u}_{0}^{\gamma, \kappa}-\mathbf{v}_{0}\right\|_{\mathbf{H}^{s}}+\left\|\theta_{0}^{\gamma, \kappa}-\rho_{0}\right\|_{H^{s}}\right) \\
& 1 \leq \delta \leq 2, \\
& \left\|\mathbf{u}^{\nu, \kappa}(t)-\mathbf{v}(t)\right\|_{\mathbf{H}^{s}}+\left\|\theta^{\gamma, \kappa}(t)-\rho(t)\right\|_{H^{s}} \\
& \leq C_{5}\left(v^{\delta / 2}+\kappa^{\delta / 2}+\left\|\mathbf{u}_{0}^{\gamma, \kappa}-\mathbf{v}_{0}\right\|_{\mathbf{H}^{s}}+\left\|\theta_{0}^{\gamma, \kappa}-\rho_{0}\right\|_{H^{s}}\right) \\
& \quad+C\left(\left\|\mathbf{u}_{0}^{v, \kappa}-\mathbf{v}_{0}\right\|_{\mathbf{H}^{s-2}}+\left\|\theta_{0}^{\nu, \kappa}-\rho_{0}\right\|_{H^{s-2}}\right)^{\delta}, \quad 0<\delta<1,
\end{aligned}
$$

where the constants $C_{4}$ and $C_{5}$ depend only on $T_{0},\left\|v_{0}\right\|_{\mathbf{H}^{s+\delta}}$, and $\left\|\rho_{0}\right\|_{H^{s+\delta}}$.

Finally, we end this section by setting some notations which will be used throughout the paper. For $p \in[1, \infty]$, $\|\cdot\|_{L^{p}}$ denotes the norm in the Lebesgue space $L^{p}\left(\mathbb{R}^{n}\right)$. We set the operator $J:=(I-\Delta)^{1 / 2}$, and for $s \in \mathbb{R}$, we denote by $H^{s, p}\left(\mathbb{R}^{n}\right)$ the nonhomogeneous Sobolev spaces with the norm defined as

$$
\|\cdot\|_{H^{s, p}}:=\left\|J^{s} \cdot\right\|_{L^{p}} .
$$

If $p=2$, for brevity, we write $H^{s}\left(\mathbb{R}^{n}\right)$ instead of $H^{s, 2}\left(\mathbb{R}^{n}\right)$. Obviously, $H^{0}\left(\mathbb{R}^{n}\right)=L^{2}\left(\mathbb{R}^{n}\right)$. In some places, we use the notation $\mathbf{H}^{s}\left(\mathbb{R}^{n} ; \mathbb{R}^{k}\right)$ to mean that this space consists of vector-valued functions $\mathbf{f}: \mathbb{R}^{n} \rightarrow \mathbb{R}^{k}$ with each component of $\mathbf{f}$ belonging to $H^{s}\left(\mathbb{R}^{n}\right)$. If there is no confusion, the spaces $\mathbf{H}^{s}\left(\mathbb{R}^{n}\right)$ and $\mathbf{H}^{s}\left(\mathbb{R}^{n} ; \mathbb{R}^{k}\right)$ will be simply denoted by $H^{s}$. For $\mathbf{f}, \mathbf{g} \in L^{2}\left(\mathbb{R}^{n} ; \mathbb{R}^{k}\right)$, we denote by $\langle\mathbf{f}, \mathbf{g}\rangle$ the usual inner product of $\mathbf{f}$ and $\mathbf{g}$; namely,

$$
\langle\mathbf{f}, \mathbf{g}\rangle:=\int_{\mathbb{R}^{n}} \mathbf{f}(\mathbf{x}) \cdot \mathbf{g}(\mathbf{x}) d \mathbf{x} .
$$


For any Banach space $\mathbf{B}$, the space $L^{p}(0, T ; \mathbf{B})$ consists of all strongly measurable functions $\mathbf{u}:[0, T] \rightarrow \mathbf{B}$ equipped with the norm

$$
\|\mathbf{u}\|_{L^{p}(0, T ; \mathbf{B})}:=\left(\int_{0}^{T}\|\mathbf{u}(t)\|_{\mathbf{B}}^{P} d t\right)^{1 / p}<\infty
$$

for $1 \leq p<\infty$, and

$$
\|\mathbf{u}\|_{L^{\infty}(0, T ; \mathbf{B})}=\text { ess } \sup _{t \in[0, T]}\|\mathbf{u}(t)\|_{\mathbf{B}}<\infty
$$

And the space $C([0, T] ; \mathbf{B})$ denotes the set of continuous functions $\mathbf{u}:[0, T] \rightarrow \mathbf{B}$ with

$$
\|\mathbf{u}\|_{C([0, T] ; \mathbf{B})}=\max _{t \in[0, T]}\|\mathbf{u}(t)\|_{\mathbf{B}}<\infty .
$$

In this paper, the letter $C$ is a generic constant and its value may change at each appearance. Moreover, every $C$ is independent of the parameters $\nu$ and $\kappa$.

\section{Proof of Theorem 1}

In this section, we present the proof of Theorem 1. To this goal, we need the following calculus inequality, the proof of which can be found in $[18,19]$.

Lemma 3. Assume that $s>0$ and $p \in(1,+\infty)$. If $f, g \in$ $\mathcal{S}\left(\mathbb{R}^{n}\right)$, the Schwartz class, then

$$
\begin{aligned}
& \left\|J^{s}(f g)-f\left(J^{s} g\right)\right\|_{L^{p}} \\
& \quad \leq C\left(\|\nabla f\|_{L^{p_{1}}}\|g\|_{H^{-1, p_{2}}}+\|f\|_{H^{s p_{3}}}\|g\|_{L^{p_{4}}}\right), \\
& \left\|J^{s}(f g)\right\|_{L^{p}} \leq C\left(\|f\|_{L^{p_{1}}}\|g\|_{H^{s p_{2}}}+\|f\|_{H^{s, p_{3}}}\|g\|_{L^{p^{p^{*}}}}\right)
\end{aligned}
$$

with $p_{2}, p_{3} \in(1,+\infty)$ such that

$$
\frac{1}{p}=\frac{1}{p_{1}}+\frac{1}{p_{2}}=\frac{1}{p_{3}}+\frac{1}{p_{4}}
$$

Of course, Lemma 3 also holds when $f$ and $g$ are replaced by vector-valued functions. Using (15) and (16), we have the following result.

Lemma 4. Let $s>1+(n / 2)$. Then,

(1) for any $\mathbf{f}, \mathbf{g} \in \mathbf{H}^{s}\left(\mathbb{R}^{n} ; \mathbb{R}^{n}\right) \cap \mathbf{H}^{r}\left(\mathbb{R}^{n} ; \mathbb{R}^{n}\right)$ with $\nabla \cdot \mathbf{f}=0$, there hold

$$
\begin{aligned}
& \left|\left\langle J^{r}((\mathbf{f} \cdot \nabla) \mathbf{g}), J^{r} \mathbf{g}\right\rangle\right| \leq C\|\mathbf{f}\|_{H^{s}}\|\mathbf{g}\|_{H^{r}}^{2}, \quad 1<r \leq s, \\
& \left|\left\langle J^{r}((\mathbf{f} \cdot \nabla) \mathbf{g}), J^{r} \mathbf{g}\right\rangle\right| \\
& \quad \leq C\|\mathbf{f}\|_{H^{s}}\|\mathbf{g}\|_{H^{r}}^{2}+C\|\mathbf{f}\|_{H^{r}}\|\mathbf{g}\|_{H^{r}}\|\mathbf{g}\|_{H^{s}}, \quad r>s ;
\end{aligned}
$$

(2) for any $\mathbf{f} \in \mathbf{H}^{s}\left(\mathbb{R}^{n} ; \mathbb{R}^{n}\right), \mathbf{g}, \mathbf{h} \in \mathbf{H}^{r}\left(\mathbb{R}^{n} ; \mathbb{R}^{n}\right)$ with $r \in$ $[0, s-1]$, there holds

$$
\left|\left\langle J^{r}((\mathbf{g} \cdot \nabla) \mathbf{f}), J^{r} \mathbf{h}\right\rangle\right| \leq C\|\mathbf{f}\|_{H^{s}}\|\mathbf{g}\|_{H^{r}}\|\mathbf{h}\|_{H^{r}} .
$$

Proof. Using the divergence free condition and the communicator estimate (15), one sees

$$
\begin{aligned}
& \left|\left\langle J^{r}((\mathbf{f} \cdot \nabla) \mathbf{g}), J^{r} \mathbf{g}\right\rangle\right| \\
& \quad=\left|\left\langle J^{r}((\mathbf{f} \cdot \nabla) \mathbf{g})-\left(\mathbf{f} \cdot J^{r} \nabla\right) \mathbf{g}, J^{r} \mathbf{g}\right\rangle\right| \\
& \quad \leq C\left(\|\nabla \mathbf{f}\|_{L^{\infty}}\left\|J^{r-1} \nabla \mathbf{g}\right\|_{L^{2}}+\left\|J^{r} \mathbf{f}\right\|_{L^{p}}\|\nabla \mathbf{g}\|_{L^{q}}\right)\left\|J^{r} \mathbf{g}\right\|_{L^{2}} \\
& \quad \leq C\left(\|\mathbf{f}\|_{H^{s}}\|\mathbf{g}\|_{H^{r}}+\|\mathbf{f}\|_{H^{r, p}}\|\mathbf{g}\|_{H^{1, q}}\right)\|\mathbf{g}\|_{H^{r}},
\end{aligned}
$$

where $p$ and $q$ satisfy $1 / p+1 / q=1 / 2(1<p<\infty)$. Since $s>1+(n / 2)$ and $r \in(1, s]$, we can always choose $p$, $q$ such that $H^{s} \hookrightarrow H^{r, p}$ and $H^{r-1} \hookrightarrow L^{q}$. In the case $r>s$, we choose $p=2$ and $q=\infty$. Hence, the estimates (18) and (19) follow immediately. For the estimate (20), the case $r=0$ is treated by the Hölder inequality, and for $r>0$, we use (16); then,

$$
\begin{aligned}
& \left|\left\langle J^{r}((\mathbf{g} \cdot \nabla) \mathbf{f}), J^{r} \mathbf{h}\right\rangle\right| \\
& \quad \leq C\left(\left\|J^{r} \mathbf{g}\right\|_{L^{2}}\|\nabla \mathbf{f}\|_{L^{\infty}}+\|\mathbf{g}\|_{L^{p}}\left\|J^{r} \nabla \mathbf{f}\right\|_{L^{q}}\right)\left\|J^{r} \mathbf{h}\right\|_{L^{2}} \\
& \quad \leq C\left(\|\mathbf{g}\|_{H^{r}}\|\mathbf{f}\|_{H^{s}}+\|\mathbf{g}\|_{L^{p}}\|\mathbf{f}\|_{H^{r+1, q}}\right)\|\mathbf{h}\|_{H^{r}} .
\end{aligned}
$$

With the condition, we can choose $p$ and $q$ satisfying $H^{r} \hookrightarrow$ $L^{p}, H^{s} \hookrightarrow H^{r+1, q}$, and $1 / p+1 / q=1 / 2(1<p<\infty)$, and thus (20) follows.

To prove Theorem 1, we first establish the uniform bounds for the solutions of (1) with the bound independent of $v$ and $\kappa$.

Lemma 5. With the same hypotheses as Theorem 1, then there exist $T>0$ and $C>0$ such that for sufficiently small $\nu, \kappa>0$, there holds

$$
\left\|\mathbf{u}^{\nu, \mathcal{K}}\right\|_{C\left([0, T] ; H^{s}\right)}+\left\|\theta^{\nu, \mathcal{\kappa}}\right\|_{C\left([0, T] ; H^{s}\right)} \leq C
$$

with $T$ and $C$ both depending only on the $H^{s}$ norm of $\left(\mathbf{v}_{0}, \rho_{0}\right)$ and not depending on $v$ and $\kappa$.

Proof. From the first equation of (1), we have

$$
\begin{gathered}
\left(J^{s} \mathbf{u}^{\nu, \kappa}\right)_{t}+J^{s}\left[\left(\mathbf{u}^{\nu, \kappa} \cdot \nabla\right) \mathbf{u}^{\nu, \kappa}\right]+\nabla J^{s} p^{\nu, \kappa} \\
=v \Delta J^{s} \mathbf{u}^{\nu, \kappa}+J^{s} \theta^{\nu, \kappa} \mathbf{e}_{2} .
\end{gathered}
$$

Multiplying this equation by $J^{s} \mathbf{u}^{\nu, \mathcal{K}}$ and integrating the result, while noting that

$$
\begin{gathered}
\int_{\mathbb{R}^{2}} \nabla J^{s} p^{\nu, \kappa} \cdot J^{\mathcal{s}} \mathbf{u}^{\nu, \kappa} d x=-\int_{\mathbb{R}^{2}} J^{s} p^{\nu, \kappa} \cdot J^{\mathcal{S}}\left(\nabla \cdot \mathbf{u}^{\nu, \kappa}\right) d x=0, \\
\left|\int_{\mathbb{R}^{2}} J^{s}\left[\left(\mathbf{u}^{\nu, \kappa} \cdot \nabla\right) \mathbf{u}^{\nu, \kappa}\right] \cdot J^{s} \mathbf{u}^{\nu, \kappa} d x\right| \leq C\left\|J^{s} \mathbf{u}^{\nu, \kappa}\right\|_{L^{2}}^{3},
\end{gathered}
$$

where we have used the estimate (18) in the previous inequality, then we obtain

$$
\begin{aligned}
& \frac{1}{2} \frac{d}{d t}\left\|J^{s} \mathbf{u}^{\nu, \kappa}\right\|_{L^{2}}^{2}+\nu\left\|\nabla J^{s} \mathbf{u}^{\nu, \kappa}\right\|_{L^{2}}^{2} \\
& \quad \leq C\left\|J^{s} \mathbf{u}^{\nu, \kappa}\right\|_{L^{2}}^{3}+\left\|J^{s} \mathbf{u}^{\nu, \kappa}\right\|_{L^{2}}\left\|J^{s} \theta^{\nu, \kappa}\right\|_{L^{2}}
\end{aligned}
$$


which gives

$$
\frac{d}{d t}\left\|\mathbf{u}^{\nu, \kappa}\right\|_{H^{s}} \leq C\left\|\mathbf{u}^{\nu, \kappa}\right\|_{H^{s}}^{2}+\left\|\theta^{\nu, \kappa}\right\|_{H^{s}} .
$$

Using the same argument to the second equation of (1), we can obtain

$$
\frac{1}{2} \frac{d}{d t}\left\|J^{s} \theta^{\gamma, \kappa}\right\|_{L^{2}}^{2}+\kappa\left\|\nabla J^{s} \theta^{\gamma, \kappa}\right\|_{L^{2}}^{2} \leq C\left\|J^{s} \mathbf{u}^{\gamma, \kappa}\right\|_{L^{2}}\left\|J^{s} \theta^{\gamma, \kappa}\right\|_{L^{2}}^{2} .
$$

Then, we have

$$
\frac{d}{d t}\left\|\theta^{\nu, \kappa}\right\|_{H^{s}} \leq C\left\|\mathbf{u}^{\nu, \kappa}\right\|_{H^{s}}\left\|\theta^{\gamma, \kappa}\right\|_{H^{s}}
$$

Hence, it concludes from the estimates (27) and (29) that

$$
\begin{aligned}
& \frac{d}{d t} \varphi^{\nu, \kappa}(t) \leq C\left(\varphi^{\nu, \kappa}(t)\right)^{2}, \\
& \varphi^{\nu, \kappa}(t):=\left\|\mathbf{u}^{\nu, \kappa}(t)\right\|_{H^{s}}+\left\|\theta^{\nu, \kappa}(t)\right\|_{H^{s}}+1 .
\end{aligned}
$$

Solving this ODE gives

$$
\varphi^{\nu, \kappa}(t) \leq \frac{\varphi^{\nu, \kappa}(0)}{1-C \varphi^{\nu, \kappa}(0) t} .
$$

Since (3) holds, we may assume $\varphi^{\nu, \kappa}(0) \leq C_{0}$ for small $\nu$ and $\kappa$ that here, $C_{0}$ depends only on $\left\|\mathbf{v}_{0}\right\|_{H^{s}}$ and $\left\|\rho_{0}\right\|_{H^{s}}$. Hence, we finally arrive at

$$
\varphi^{\nu, \kappa}(t) \leq \frac{C_{0}}{1-C C_{0} t} .
$$

The estimate (23) follows from the previous inequality provided that we select $T$ such that $T<1 / C C_{0}$ (e.g., we can choose $T=1 / 2 C C_{0}$ ). The proof of Lemma 5 is complete.

Remark 6. From the proof of Lemma 5, we also see the solution of system (2) satisfying

$$
\|\mathbf{v}\|_{C\left([0, T] ; H^{s}\right)}+\|\rho\|_{C\left([0, T] ; H^{s}\right)} \leq C,
$$

where $T$ and $C$ are the same as (23).

Remark 7. For fixed $T_{0} \in\left(0, T^{*}\right)$, without loss of generality, we may assume that the time $T$ determined by Lemma 5 satisfies $T<T_{0}$. Indeed, as will be seen in the proof of Theorem 1, no matter how small the $T$ is, we can always use bootstrap argument to extend the interval $[0, T]$ into our desired time interval $\left[0, T_{0}\right]$.

In the following, we define $\mathbf{w}^{\nu, \kappa}:=\mathbf{u}^{\nu, \kappa}-\mathbf{v}$ and $\chi^{\nu, \kappa}:=$ $\theta^{\nu, \kappa}-\rho$. Considering the equations, for $\left(\mathbf{u}^{\nu, \kappa}, \theta^{\nu, \kappa}\right)$ and $(\mathbf{v}, \rho)$, we see $\left(\mathbf{w}^{\nu, \kappa}, \chi^{\gamma, \kappa}\right)$ satisfying

$$
\begin{gathered}
\mathbf{w}_{t}^{\gamma, \kappa}+(\mathbf{v} \cdot \nabla) \mathbf{w}^{\nu, \kappa}+\left(\mathbf{w}^{\nu, \kappa} \cdot \nabla\right) \mathbf{u}^{\nu, \kappa}+\nabla\left(p^{\nu, \kappa}-\pi\right) \\
=v \Delta \mathbf{w}^{\nu, \kappa}+\nu \Delta \mathbf{v}+\chi^{\nu, \kappa} \mathbf{e}_{2}, \\
\chi_{t}^{\nu, \kappa}+(\mathbf{v} \cdot \nabla) \chi^{\nu, \kappa}+\left(\mathbf{w}^{\nu, \kappa} \cdot \nabla\right) \theta^{\nu, \kappa}=\kappa \Delta \chi^{\nu, \kappa}+\kappa \Delta \rho, \\
\nabla \cdot \mathbf{w}^{\nu, \kappa}=0,
\end{gathered}
$$

$$
\begin{aligned}
& \left.\mathbf{w}^{\nu, \kappa}\right|_{t=0}=\mathbf{u}_{0}^{\nu, \kappa}-\mathbf{v}_{0}, \\
& \left.\chi^{\nu, \kappa}\right|_{t=0}=\theta_{0}^{\nu, \kappa}-\rho_{0} .
\end{aligned}
$$

For the sake of convenience, we often omit the superscripts $\nu$ and $\kappa$ in the succeeding arguments; hence, $\mathbf{w}$ means $\mathbf{w}^{\nu, \kappa}, \theta$ stands for $\theta^{\nu, \kappa}$, and so on.

Proof of Theorem 1. We split the proof into several steps.

Step 1. We first show that (4) holds on $[0, T]$. By using (18) and (20) with $r=s-2$, we can obtain the $H^{s-2}$ energy for $\mathbf{w}$ as

$$
\begin{aligned}
\frac{1}{2} \frac{d}{d t}\|\mathbf{w}\|_{H^{s-2}}^{2}+\nu\|\nabla \mathbf{w}\|_{H^{s-2}}^{2} & \\
\leq & C\left(\|\mathbf{v}\|_{H^{s}}+\|\mathbf{u}\|_{H^{s}}\right)\|\mathbf{w}\|_{H^{s-2}}^{2} \\
& +\nu\|\Delta \mathbf{v}\|_{H^{s-2}}\|\mathbf{w}\|_{H^{s-2}}+\|\chi\|_{H^{s-2}}\|\mathbf{w}\|_{H^{s-2}} \\
\leq & C\|\mathbf{w}\|_{H^{s-2}}^{2}+C \nu\|\mathbf{w}\|_{H^{s-2}}+\|\chi\|_{H^{s-2}}\|\mathbf{w}\|_{H^{s-2}},
\end{aligned}
$$

where we have used the uniform estimates (23) and (33) in the last step. Hence, we get

$$
\frac{d}{d t}\|\mathbf{w}\|_{H^{s-2}} \leq C\|\mathbf{w}\|_{H^{s-2}}+C v+\|\chi\|_{H^{s-2}} .
$$

Similarly, the $H^{s-2}$ energy estimate for $\chi$ can be written as

$$
\begin{aligned}
\frac{1}{2} \frac{d}{d t} & \|\chi\|_{H^{s-2}}^{2}+\kappa\|\nabla \chi\|_{H^{s-2}}^{2} \\
\leq & C\left(\|\Delta \mathbf{v}\|_{H^{s}}\|\chi\|_{H^{s-2}}^{2}+\|\mathbf{w}\|_{H^{s-2}}\|\theta\|_{H^{s}}\|\chi\|_{H^{s-2}}\right) \\
& +\kappa\|\Delta \rho\|_{H^{s-2}}\|\chi\|_{H^{s-2}} \\
\leq & C\|\chi\|_{H^{s-2}}^{2}+C\|\mathbf{w}\|_{H^{s-2}}\|\chi\|_{H^{s-2}}+C \kappa\|\chi\|_{H^{s-2}}
\end{aligned}
$$

which gives

$$
\frac{d}{d t}\|\chi\|_{H^{s-2}} \leq C\|\chi\|_{H^{s-2}}+C\|\mathbf{w}\|_{H^{s-2}}+C \kappa .
$$

Therefore, one has

$$
\begin{gathered}
\frac{d}{d t} \phi_{1}(t) \leq C \phi_{1}(t)+C \nu+C \kappa, \\
\phi_{1}(t):=\|\mathbf{w}(t)\|_{H^{s-2}}+\|\chi(t)\|_{H^{s-2}} .
\end{gathered}
$$

Then, the Gronwall inequality yields that for all $t \in[0, T](C$ $\left.\left[0, T_{0}\right]\right)$,

$$
\phi_{1}(t) \leq e^{C t} \phi_{1}(0)+C(\nu+\kappa) t e^{C t} \leq C_{1}\left(\phi_{1}(0)+\nu+\kappa\right),
$$

where $C_{1}:=e^{C T_{0}}\left(1+C T_{0}\right)$ with $C$ depending on $\left\|v_{0}\right\|_{H^{s}}$ and $\left\|\rho_{0}\right\|_{H^{s}}$. 
Step 2. We show that (5) holds on $[0, T]$. Applying the estimates (18) and (20) with $r=s_{1}$, then we can easily obtain the $H^{s_{1}}$ energy for $\mathbf{w}$ as

$$
\begin{aligned}
\frac{1}{2} \frac{d}{d t} & \|\mathbf{w}\|_{H^{s_{1}}}^{2}+\nu\|\nabla \mathbf{w}\|_{H^{s_{1}}}^{2} \\
\leq & C\left(\|\mathbf{v}\|_{H^{s}}+\|\mathbf{u}\|_{H^{s}}\right)\|\mathbf{w}\|_{H^{s_{1}}}^{2} \\
& +\nu\|\nabla \mathbf{v}\|_{H^{s-1}}\|\nabla \mathbf{w}\|_{H^{2 s_{1}-s+1}}+\|\chi\|_{H^{s_{1}}}\|\mathbf{w}\|_{H^{s_{1}}} \\
\leq & C\|\mathbf{w}\|_{H^{s_{1}}}^{2}+C \nu\|\nabla \mathbf{w}\|_{H^{2 s_{1}-s+1}}+\|\chi\|_{H^{s_{1}}}\|\mathbf{w}\|_{H^{s_{1}}} .
\end{aligned}
$$

Since $s_{1} \in(s-2, s-1]$, we have $s_{1}-1<2 s_{1}-s+1 \leq$ $s_{1}$. By the Gagliardo-Nirenberg interpolation inequality and Young's inequality, we have

$$
\begin{aligned}
& C\|\nabla \mathbf{w}\|_{H^{2 s_{1}-s+1}} \\
& \quad \leq C\|\nabla \mathbf{w}\|_{H^{s_{1}-1}}^{s-s_{1}-1}\|\nabla \mathbf{w}\|_{H^{s_{1}}}^{2-s+s_{1}} \\
& \quad \leq C\|\mathbf{w}\|_{H^{s_{1}}}^{2\left(s-s_{1}-1\right) /\left(s-s_{1}\right)}+\|\nabla \mathbf{w}\|_{H^{s_{1}}}^{2} .
\end{aligned}
$$

Inserting this inequality into (41) and using Young's inequality again, we thus get

$$
\begin{aligned}
\frac{1}{2} \frac{d}{d t} & \|\nabla \mathbf{w}\|_{H^{s_{1}}}^{2} \\
\leq & C\|\nabla \mathbf{w}\|_{H^{s_{1}}}^{2}+C \nu\|\mathbf{w}\|_{H^{s_{1}}}^{2\left(s-s_{1}-1\right) /\left(s-s_{1}\right)} \\
& +\|\chi\|_{H^{s_{1}}}\|\mathbf{w}\|_{H^{s_{1}}} \\
\leq & C\|\mathbf{w}\|_{H^{s_{1}}}^{2}+C \nu^{s-s_{1}}+C\|\chi\|_{H^{s_{1}}}^{2} .
\end{aligned}
$$

With similar argument as abovementioned, we can also obtain the $H^{s_{1}}$ energy for $\chi$ as

$$
\frac{1}{2} \frac{d}{d t}\|\chi\|_{H^{s_{1}}}^{2} \leq C\|\chi\|_{H^{s_{1}}}^{2}+C\|\mathbf{w}\|_{H^{s_{1}}}^{2}+C \kappa^{s-s_{1}} .
$$

The previous two estimates give

$$
\begin{aligned}
& \frac{d}{d t} \phi_{2}(t) \leq C \phi_{2}(t)+C \nu^{s-s_{1}}+C \kappa^{s-s_{1}}, \\
& \phi_{2}(t):=\|\mathbf{w}(t)\|_{H^{s_{1}}}^{2}+\|\chi(t)\|_{H^{s_{1}}}^{2},
\end{aligned}
$$

and by the Gronwall inequality we get

$$
\begin{array}{r}
\phi_{2}(t) \leq e^{C t} \phi_{2}(0)+C\left(\nu^{s-s_{1}}+\kappa^{s-s_{1}}\right) t e^{C t} \\
\leq C_{2}\left(\phi_{2}(0)+\nu^{s-s_{1}}+\kappa^{s-s_{1}}\right), \\
\forall t \in[0, T],
\end{array}
$$

which implies that the estimate (5) holds on $[0, T]$ with $C_{2}:=$ $e^{C T_{0}}\left(1+C T_{0}\right)$.

Step 3. To prove (7), we need to regularize the initial data. Define $\psi(x) \in C_{0}^{\infty}\left(\mathbb{R}^{2}\right)$ by

$$
\psi(x)= \begin{cases}c_{0} \exp \left(-\frac{1}{1-|x|^{2}}\right), & |x|<1, \\ 0, & |x| \geq 1,\end{cases}
$$

where the constant $c_{0}$ is selected so that $\int_{\mathbb{R}^{2}} \psi(x) d x=1$. Let $\psi_{\epsilon}(x)=\epsilon^{-2} \rho\left(\epsilon^{-1} x\right)$, and define the mollification $\mathscr{J}_{\epsilon} f$ of $f \epsilon$ $L_{\text {loc }}^{1}\left(\mathbb{R}^{2}\right)$ by $\left(\mathscr{J}_{\epsilon} f\right)(x)=\left(\psi_{\epsilon} * f\right)(x)$. By this definition, one can see $\mathscr{J}_{\epsilon} f \in C^{\infty}$; moreover, if $f \in H^{s}$, we have $\mathscr{J}_{\epsilon} f \rightarrow f$ in $H^{s}$ as $\epsilon \rightarrow 0$ and

$$
\begin{gathered}
\operatorname{Lim}_{\epsilon \rightarrow 0}\left\|\mathscr{J}_{\epsilon} f-f\right\|_{s-k} \leq C_{s k} \epsilon^{k}\|f\|_{s^{\prime}} \\
\left\|\mathscr{J}_{\epsilon} f\right\|_{s+k} \leq \frac{C_{s k}}{\epsilon^{k}}\|f\|_{s} .
\end{gathered}
$$

For the proof of these properties, see Lemma 3.5 in [20]. Now let $\left(\mathbf{v}^{\epsilon}, \rho^{\epsilon}\right)$ be the solution of the inviscid system (2) with initial data $\left(\mathscr{J}_{\epsilon} \mathbf{v}_{0}, \mathscr{J}_{\epsilon} \rho_{0}\right)$; namely, $\left(\mathbf{v}^{\epsilon}, \rho^{\epsilon}\right)$ solves the equations

$$
\begin{gathered}
\mathbf{v}_{t}^{\epsilon}+\left(\mathbf{v}^{\epsilon} \cdot \nabla\right) \mathbf{v}^{\epsilon}+\nabla \pi^{\epsilon}=\rho^{\epsilon} \mathbf{e}_{2}, \\
\rho_{t}^{\epsilon}+\left(\mathbf{v}^{\epsilon} \cdot \nabla\right) \rho^{\epsilon}=0, \\
\nabla \cdot \mathbf{v}^{\epsilon}=0, \\
\left.\mathbf{v}^{\epsilon}\right|_{t=0}=\mathscr{J}_{\epsilon} \mathbf{v}_{0},\left.\rho^{\epsilon}\right|_{t=0}=\mathscr{J}_{\epsilon} \rho_{0} .
\end{gathered}
$$

So, the $H^{s}$ energy for $\mathbf{v}^{\epsilon}$ and $\rho^{\epsilon}$ can be written as

$$
\begin{aligned}
& \frac{1}{2} \frac{d}{d t}\left\|\mathbf{v}^{\epsilon}\right\|_{H^{s}}^{2} \leq C\left\|\mathbf{v}^{\epsilon}\right\|_{H^{s}}^{3}+\left\|\mathbf{v}^{\epsilon}\right\|_{H^{s}}\left\|\rho^{\epsilon}\right\|_{H^{s}}, \\
& \frac{1}{2} \frac{d}{d t}\left\|\rho^{\epsilon}\right\|_{H^{s}}^{2} \leq C\left\|\mathbf{v}^{\epsilon}\right\|_{H^{s}}\left\|\rho^{\epsilon}\right\|_{H^{s}}^{2} .
\end{aligned}
$$

With the same discussion as Lemma 5, we know that there exist $T^{\prime}>0$ and $C>0$ both only depending on the $H^{s}$ norm of $\left(\mathbf{v}_{0}, \rho_{0}\right)$ such that

$$
\left\|\mathbf{v}^{\epsilon}\right\|_{C\left(\left[0, T^{\prime}\right] ; H^{s}\right)}+\left\|\rho^{\epsilon}\right\|_{C\left(\left[0, T^{\prime}\right] ; H^{s}\right)} \leq C .
$$

Moreover, taking the $H^{s+k}$ energy for $\mathbf{v}^{\epsilon}$ and $\rho^{\epsilon}$ and using (19), then for $k \in \mathbb{N}^{+}$,

$$
\begin{aligned}
\frac{1}{2} \frac{d}{d t}\left\|\mathbf{v}^{\epsilon}\right\|_{H^{s+k}}^{2} & \\
\leq & C\left\|\mathbf{v}^{\epsilon}\right\|_{H^{s}}\left\|\mathbf{v}^{\epsilon}\right\|_{H^{s+k}}^{2} \\
& +\left\|\mathbf{v}^{\epsilon}\right\|_{H^{s+k}}\left\|\rho^{\epsilon}\right\|_{H^{s+k}} \leq C\left\|\mathbf{v}^{\epsilon}\right\|_{H^{s+k}}^{2} \\
& +C\left\|\rho^{\epsilon}\right\|_{H^{s+k}}^{2},
\end{aligned}
$$

$$
\begin{aligned}
\frac{1}{2} \frac{d}{d t}\left\|\rho^{\epsilon}\right\|_{H^{s+k}}^{2} & \\
\leq & C\left\|\mathbf{v}^{\epsilon}\right\|_{H^{s}}\left\|\rho^{\epsilon}\right\|_{H^{s+k}}^{2} \\
& +C\left\|\mathbf{v}^{\epsilon}\right\|_{H^{s+k}}\left\|\rho^{\epsilon}\right\|_{H^{s+k}}\left\|\rho^{\epsilon}\right\|_{H^{s}} \\
\leq & C\left\|\mathbf{v}^{\epsilon}\right\|_{H^{s+k}}^{2}+C\left\|\rho^{\epsilon}\right\|_{H^{s+k}}^{2} .
\end{aligned}
$$

Using (48), we deduce from the Previous energy estimate that

$$
\left\|\mathbf{v}^{\epsilon}\right\|_{C\left(\left[0, T^{\prime}\right] ; H^{s+k}\right)}+\left\|\rho^{\epsilon}\right\|_{C\left(\left[0, T^{\prime}\right] ; H^{s+k}\right)} \leq \frac{C e^{C T_{0}}}{\epsilon^{k}}, \quad k \in \mathbb{N}^{+}
$$

where $C$ depends only on $k,\left\|\mathbf{v}_{0}\right\|_{H^{s}}$, and $\left\|\rho_{0}\right\|_{H^{s}}$. Without loss of generality, in the following, we may assume $T^{\prime}=T$, where that $T$ is determined by Lemma 5 . 
Step 4. Set $\widetilde{\mathbf{v}}^{\epsilon}=\mathbf{v}^{\epsilon}-\mathbf{v}$ and $\widetilde{\rho}^{\epsilon}=\rho^{\epsilon}-\rho$; then, from (2) and (49), we know that $\left(\widetilde{\mathbf{v}}^{\epsilon}, \widetilde{\rho}^{\epsilon}\right)$ satisfies

$$
\begin{aligned}
& \widetilde{\mathbf{v}}_{t}^{\epsilon}+(\mathbf{v} \cdot \nabla) \widetilde{\mathbf{v}}^{\epsilon}+\left(\widetilde{\mathbf{v}}^{\epsilon} \cdot \nabla\right) \mathbf{v}^{\epsilon}+\nabla\left(\pi^{\epsilon}-\pi\right)=\widetilde{\rho}^{\epsilon} \mathbf{e}_{2}, \\
& \widetilde{\rho}_{t}^{\epsilon}+(\mathbf{v} \cdot \nabla) \widetilde{\rho}^{\epsilon}+\left(\widetilde{\mathbf{v}}^{\epsilon} \cdot \nabla\right) \rho^{\epsilon}=0 .
\end{aligned}
$$

Using (18) with $r=s$, we have

$$
\left|\left\langle J^{s}\left(\left(\mathbf{v}^{\epsilon} \cdot \nabla\right) \widetilde{\mathbf{v}}^{\epsilon}\right), J^{s} \widetilde{\mathbf{v}}^{\epsilon}\right\rangle\right| \leq C\left\|\mathbf{v}^{\epsilon}\right\|_{H^{s}}\left\|\widetilde{\mathbf{v}}^{\epsilon}\right\|_{H^{s}}^{2}
$$

By the Cauchy-Schwarz inequality and (16), one has

$$
\begin{aligned}
\left|\left\langle J^{s}\left(\left(\widetilde{\mathbf{v}}^{\epsilon} \cdot \nabla\right) \mathbf{v}^{\epsilon}\right), J^{s} \widetilde{\mathbf{v}}^{\epsilon}\right\rangle\right| & \\
\leq C & \left(\left\|J^{s} \widetilde{\mathbf{v}}^{\epsilon}\right\|_{L^{2}}\left\|\nabla \mathbf{v}^{\epsilon}\right\|_{L^{\infty}}\right. \\
& \left.+\left\|\widetilde{\mathbf{v}}^{\epsilon}\right\|_{L^{\infty}}\left\|J^{s} \nabla \mathbf{v}^{\epsilon}\right\|_{L^{2}}\right)\left\|J^{s} \widetilde{\mathbf{v}}^{\epsilon}\right\|_{L^{2}} \\
\leq C & \left(\left\|\widetilde{\mathbf{v}}^{\epsilon}\right\|_{H^{s}}\left\|\mathbf{v}^{\epsilon}\right\|_{H^{s}}\right. \\
& \left.+\left\|\widetilde{\mathbf{v}}^{\epsilon}\right\|_{L^{\infty}}\left\|\mathbf{v}^{\epsilon}\right\|_{H^{s+1}}\right)\left\|\widetilde{\mathbf{v}}^{\epsilon}\right\|_{H^{s}} .
\end{aligned}
$$

With these two estimates, it is easy to obtain the following $H^{s}$ energy estimate for $\widetilde{\mathbf{v}}^{\epsilon}$ :

$$
\begin{aligned}
\frac{1}{2} \frac{d}{d t}\left\|\widetilde{\mathbf{v}}^{\epsilon}\right\|_{H^{s}}^{2} & \\
\leq & C\left(\left\|\mathbf{v}^{\epsilon}\right\|_{H^{s}}+\|\mathbf{v}\|_{H^{s}}\right)\left\|\widetilde{\mathbf{v}}^{\epsilon}\right\|_{H^{s}}^{2} \\
& +C\left\|\widetilde{\mathbf{v}}^{\epsilon}\right\|_{L^{\infty}}\left\|\mathbf{v}^{\epsilon}\right\|_{H^{s+1}}\left\|\widetilde{\mathbf{v}}^{\epsilon}\right\|_{H^{s}}+\left\|\widetilde{\rho}^{\epsilon}\right\|_{H^{s}}\left\|\widetilde{\mathbf{v}}^{\epsilon}\right\|_{H^{s}} \\
\leq & C\left\|\widetilde{\mathbf{v}}^{\epsilon}\right\|_{H^{s}}^{2}+C e^{C T_{0}} \epsilon^{-1}\left\|\widetilde{\mathbf{v}}^{\epsilon}\right\|_{L^{\infty}}\left\|\widetilde{\mathbf{v}}^{\epsilon}\right\|_{H^{s}} \\
& +\left\|\widetilde{\rho}^{\epsilon}\right\|_{H^{s}}\left\|\widetilde{\mathbf{v}}^{\epsilon}\right\|_{H^{s}},
\end{aligned}
$$

where we have used (54) and the uniform estimate (51) in the last step. Similarly, we can obtain the following $H^{s}$ energy estimate for $\tilde{\rho}^{\epsilon}$ :

$$
\begin{aligned}
& \frac{1}{2} \frac{d}{d t}\left\|\widetilde{\rho}^{\epsilon}\right\|_{H^{s}}^{2} \\
& \leq C\left(\|\mathbf{v}\|_{H^{s}}+\left\|\widetilde{\rho}^{\epsilon}\right\|_{H^{s}}\right)\left\|\widetilde{\rho}^{\epsilon}\right\|_{H^{s}}^{2} \\
& \quad+C\left\|\widetilde{\mathbf{v}}^{\epsilon}\right\|_{L^{\infty}}\left\|\rho^{\epsilon}\right\|_{H^{s+1}}\left\|\widetilde{\rho}^{\epsilon}\right\|_{H^{s}} \\
& \leq C\left\|\widetilde{\rho}^{\epsilon}\right\|_{H^{s}}^{2}+C e^{C T_{0}} \epsilon^{-1}\left\|\widetilde{\mathbf{v}}^{\epsilon}\right\|_{L^{\infty}}\left\|\widetilde{\rho}^{\epsilon}\right\|_{H^{s}} .
\end{aligned}
$$

Now, we have to estimate $\left\|\widetilde{\mathbf{v}}^{\epsilon}\right\|_{L^{\infty}}$. From (55), we take the $H^{s-2}$ energy for $\left(\widetilde{\mathbf{v}}^{\epsilon}, \widetilde{\rho}^{\epsilon}\right)$ and obtain (using (18) and (20))

$$
\begin{aligned}
& \frac{1}{2} \frac{d}{d t}\left\|\widetilde{\mathbf{v}}^{\epsilon}\right\|_{H^{s-2}}^{2} \\
& \quad \leq C\left\|\widetilde{\mathbf{v}}^{\epsilon}\right\|_{H^{s-2}}^{2}+C\left\|\widetilde{\rho}^{\epsilon}\right\|_{H^{s-2}}\left\|\widetilde{\mathbf{v}}^{\epsilon}\right\|_{H^{s-2}} \\
& \frac{1}{2} \frac{d}{d t}\left\|\widetilde{\rho}^{\epsilon}\right\|_{H^{s-2}}^{2} \\
& \quad \leq C\left\|\widetilde{\rho}^{\epsilon}\right\|_{H^{s-2}}^{2}+C\left\|\widetilde{\rho}^{\epsilon}\right\|_{H^{s-2}}\left\|\widetilde{\mathbf{v}}^{\epsilon}\right\|_{H^{s-2}}
\end{aligned}
$$

Then, the Gronwall inequality and (48) yield

$$
\begin{aligned}
& \left\|\widetilde{\mathbf{v}}^{\epsilon}(t)\right\|_{H^{s-2}}+\left\|\widetilde{\rho}^{\epsilon}(t)\right\|_{H^{s-2}} \\
& \leq e^{C T_{0}}\left(\left\|\widetilde{\mathbf{v}}^{\epsilon}(0)\right\|_{H^{s-2}}+\left\|\widetilde{\rho}^{\epsilon}(0)\right\|_{H^{s-2}}\right) \\
& \quad \leq C e^{C T_{0}} \epsilon^{2},
\end{aligned}
$$

for all $t \in[0, T]$, which in turn by Sobolev embedding theorem gives $\left\|\widetilde{\mathbf{v}}^{\epsilon}(t)\right\|_{L^{\infty}}+\left\|\widetilde{\rho}^{\epsilon}(t)\right\|_{L^{\infty}} \leq C e^{C T_{0}} \epsilon^{2}$ since $s>3$. Inserting this estimate into (58) and (59), we can see

$$
\frac{d}{d t} \phi_{3} \leq C e^{C T_{0}} \phi_{3}+C e^{C T_{0}} \epsilon, \quad \phi_{3}:=\left\|\widetilde{\mathbf{v}}^{\epsilon}\right\|_{H^{s}}+\left\|\widetilde{\rho}^{\epsilon}\right\|_{H^{s}}
$$

where we have used the relation $e^{C T_{0}} \cdot e^{C T_{0}}=e^{2 C T_{0}}=: e^{C T_{0}}$ in the last step since the value of $C$ at each appearance may be different. Hence, the Gronwall inequality gives

$$
\begin{array}{r}
\phi_{3}(t) \leq e^{C T_{0} e^{C T_{0}}}\left(1+C T_{0} e^{C T_{0}}\right)\left(\phi_{3}(0)+\epsilon\right), \\
\forall t \in[0, T] .
\end{array}
$$

Step 5. Let $\mathbf{U}^{\epsilon}=\mathbf{u}-\mathbf{v}^{\epsilon}, \Theta^{\epsilon}=\theta-\rho^{\epsilon}$, and recall that here $\mathbf{u}=\mathbf{u}^{\nu, \kappa}$ and $\theta=\theta^{\nu, \kappa}$; so, one can deduce from (2) and (55) that $\left(\mathbf{U}^{\epsilon}, \Theta^{\epsilon}\right)$ solves

$$
\begin{aligned}
\mathbf{U}_{t}^{\epsilon}+ & (\mathbf{u} \cdot \nabla) \mathbf{U}^{\epsilon}+\left(\mathbf{U}^{\epsilon} \cdot \nabla\right) \mathbf{v}^{\epsilon}+\nabla\left(p-\pi^{\epsilon}\right) \\
& =v \Delta \mathbf{U}^{\epsilon}+v \Delta \mathbf{v}^{\epsilon}+\Theta^{\epsilon} \mathbf{e}_{2}, \\
\Theta_{t}^{\epsilon}+ & (\mathbf{u} \cdot \nabla) \Theta^{\epsilon}+\left(\mathbf{U}^{\epsilon} \cdot \nabla\right) \rho^{\epsilon}=\kappa \Delta \Theta^{\epsilon}+\kappa \Delta \rho^{\epsilon} .
\end{aligned}
$$

Using the same reasonings that lead to (58), we have the $H^{s}$ energy estimate for $\mathbf{U}^{\epsilon}$ as

$$
\begin{aligned}
\frac{1}{2} \frac{d}{d t}\left\|\mathbf{U}^{\epsilon}\right\|_{H^{s}}^{2}+\nu\left\|\nabla \mathbf{U}^{\epsilon}\right\|_{H^{s}}^{2} & \\
\leq & C\left(\|\mathbf{u}\|_{H^{s}}+\left\|\mathbf{v}^{\epsilon}\right\|_{H^{s}}\right)\left\|\mathbf{U}^{\epsilon}\right\|_{H^{s}}^{2} \\
& +C\left\|\mathbf{v}^{\epsilon}\right\|_{H^{s+1}}\left\|\mathbf{U}^{\epsilon}\right\|_{L^{\infty}}\left\|\mathbf{U}^{\epsilon}\right\|_{H^{s}} \\
& +\nu\left\|\mathbf{v}^{\epsilon}\right\|_{H^{s+2}}\left\|\mathbf{U}^{\epsilon}\right\|_{H^{s}}+\left\|\Theta^{\epsilon}\right\|_{H^{s}}\left\|\mathbf{U}^{\epsilon}\right\|_{H^{s}} \\
\leq & C\left\|\mathbf{U}^{\epsilon}\right\|_{H^{s}}^{2}+C e^{C T_{0}} \epsilon^{-1}\left\|\mathbf{U}^{\epsilon}\right\|_{L^{\infty}}\left\|\mathbf{U}^{\epsilon}\right\|_{H^{s}} \\
& +C e^{C T_{0}} \nu \epsilon^{-2}\left\|\mathbf{U}^{\epsilon}\right\|_{H^{s}}+\left\|\Theta^{\epsilon}\right\|_{H^{s}}\left\|\mathbf{U}^{\epsilon}\right\|_{H^{s}},
\end{aligned}
$$

which yields

$$
\begin{gathered}
\frac{d}{d t}\left\|\mathbf{U}^{\epsilon}\right\|_{H^{s}} \leq C\left\|\mathbf{U}^{\epsilon}\right\|_{H^{s}}+C e^{C T_{0}} \epsilon^{-1}\left\|\mathbf{U}^{\epsilon}\right\|_{L^{\infty}} \\
+C e^{C T_{0}} \nu \epsilon^{-2}+\left\|\Theta^{\epsilon}\right\|_{H^{s}} .
\end{gathered}
$$

Similarly, we can obtain the $H^{s}$ energy for $\Theta^{\epsilon}$ as

$$
\begin{gathered}
\frac{d}{d t}\left\|\Theta^{\epsilon}\right\|_{H^{s}} \leq C\left\|\Theta^{\epsilon}\right\|_{H^{s}}+C\left\|\mathbf{U}^{\epsilon}\right\|_{H^{s}} \\
+C e^{C T_{0}} \epsilon^{-1}\left\|\mathbf{U}^{\epsilon}\right\|_{L^{\infty}}+C e^{C T_{0}} \kappa \epsilon^{-2} .
\end{gathered}
$$


Now, we should estimate $\left\|\mathbf{U}^{\epsilon}\right\|_{L^{\infty}}$. Note that $\mathbf{U}^{\epsilon}=\mathbf{w}-\widetilde{\mathbf{v}}^{\epsilon}$; so,

$$
\left\|\mathbf{U}^{\epsilon}\right\|_{L^{\infty}} \leq\|\mathbf{w}\|_{L^{\infty}}+\left\|\widetilde{\mathbf{v}}^{\epsilon}\right\|_{L^{\infty}} \text {. }
$$

By (40), (62), and Sobolev embedding theorem, we have

$$
\begin{aligned}
& \|\mathbf{w}\|_{L^{\infty}\left(0, T ; L^{\infty}\right)} \leq C\|\mathbf{w}\|_{L^{\infty}\left(0, T ; H^{s-2}\right)} \leq C_{1}\left(\phi_{1}(0)+\nu+\kappa\right), \\
& \left\|\widetilde{\mathbf{v}}^{\epsilon}\right\|_{L^{\infty}\left(0, T ; L^{\infty}\right)} \leq C\left\|\widetilde{\mathbf{v}}^{\epsilon}\right\|_{L^{\infty}\left(0, T ; H^{s-2}\right)} \leq C e^{C T_{0}} \epsilon^{2},
\end{aligned}
$$

which gives

$$
\begin{gathered}
\left\|\mathbf{U}^{\epsilon}\right\|_{L^{\infty}} \leq C^{*}\left(\phi_{1}(0)+\nu+\kappa+\epsilon^{2}\right), \\
C^{*}:=\max \left\{C_{1}, C e^{C T_{0}}\right\} .
\end{gathered}
$$

Inserting this estimate into (67) and (68), one has

$$
\begin{aligned}
\frac{d}{d t} \phi_{4} \leq & C \phi_{4}+C e^{C T_{0}} \epsilon^{-1}\left\|\mathbf{U}^{\epsilon}\right\|_{L^{\infty}}+C e^{C T_{0}}(\nu+\kappa) \epsilon^{-2} \\
\leq & C \phi_{4}+C e^{C T_{0}} \epsilon^{-1} C^{*}\left(\phi_{1}(0)+\nu+\kappa+\epsilon^{2}\right) \\
& +C e^{C T_{0}}(\nu+\kappa) \epsilon^{-2} \\
\leq & C^{* *} \phi_{4}+C^{* *} \epsilon+C^{* *} \epsilon^{-1} \phi_{1}(0)+C^{* *} \epsilon^{-2}(\nu+\kappa),
\end{aligned}
$$

where $\phi_{4}:=\left\|\mathbf{U}^{\epsilon}\right\|_{H^{s}}+\left\|\Theta^{\epsilon}\right\|_{H^{s}}$ and $C^{* *}:=\max \{C$, $\left.C e^{C T_{0}} C^{*}, C e^{C T_{0}}\right\}$. By the Gronwall inequality, one obtains

$$
\begin{aligned}
\phi_{4}(t) & \\
& \leq e^{C^{* *} T_{0}}\left(1+C^{* *} T_{0}\right) \\
& \times\left(\phi_{4}(0)+\epsilon+\epsilon^{-1} \phi_{1}(0)+\epsilon^{-2}(\nu+\kappa)\right) .
\end{aligned}
$$

Recall that

$$
\mathbf{w}=\mathbf{U}^{\epsilon}+\widetilde{\mathbf{v}}^{\epsilon}, \quad \chi=\Theta^{\epsilon}+\widetilde{\rho}^{\epsilon} ;
$$

therefore, it follows from (64) and (73) that

$$
\begin{aligned}
\|\mathbf{w}(t)\|_{H^{s}}+\|\chi(t)\|_{H^{s}} \leq \phi_{3}(t)+\phi_{4}(t) \\
\leq \widetilde{C}\left(\phi_{3}(0)+\phi_{4}(0)+\epsilon+\epsilon^{-1} \phi_{1}(0)+\epsilon^{-2}(\nu+\kappa)\right) \\
\leq 2 \widetilde{C}\left(\phi_{3}(0)+\|\mathbf{w}(0)\|_{H^{s}}+\|\chi(0)\|_{H^{s}}\right. \\
\left.+\epsilon+\epsilon^{-1} \phi_{1}(0)+\epsilon^{-2}(\nu+\kappa)\right)
\end{aligned}
$$

for all $t \in[0, T]$, where we use $\phi_{4}(0) \leq \phi_{1}(0)+\phi_{3}(0)$ in the last step and

$$
\widetilde{C}:=\max \left\{e^{C^{* *} T_{0}}\left(1+C^{* *} T_{0}\right), e^{C T_{0} e^{C T_{0}}}\left(1+C T_{0} e^{C T_{0}}\right)\right\} .
$$

Note that (3) gives

$$
\|\mathbf{w}(0)\|_{H^{s}}+\|\chi(0)\|_{H^{s}}=\left\|\mathbf{u}_{0}^{\nu, \kappa}-\mathbf{v}_{0}\right\|_{H^{s}}+\left\|\theta_{0}^{\nu, \kappa}-\rho_{0}\right\|_{H^{s}} \underset{\text { (77) }}{\longrightarrow} 0
$$

as $(\nu, \kappa) \rightarrow 0$, and the property of the operator $\mathscr{J}_{\epsilon}$ yields

$$
\begin{aligned}
\phi_{3}(0) & =\left\|\tilde{\mathbf{v}}^{\epsilon}(0)\right\|_{H^{s}}+\left\|\widetilde{\rho}^{\epsilon}(0)\right\|_{H^{s}} \\
& =\left\|\mathbf{v}^{\epsilon}(0)-\mathbf{v}(0)\right\|_{H^{s}}+\left\|\rho^{\epsilon}(0)-\rho(0)\right\|_{H^{s}} \\
& =\left\|\mathscr{J}_{\epsilon} \mathbf{v}_{0}-\mathbf{v}_{0}\right\|_{H^{s}}+\left\|\mathscr{J}_{\epsilon} \rho_{0}-\rho_{0}\right\|_{H^{s}} \longrightarrow 0
\end{aligned}
$$

as $\epsilon \rightarrow 0$. Now, we choose $\epsilon=\epsilon(\nu, \kappa)>0$ satisfying the following properties:

(1) $\lim _{(v, \kappa) \rightarrow 0} \epsilon=0$,

(2) $\lim _{(\nu, \kappa) \rightarrow 0} \epsilon^{-1} \phi_{1}(0)=\lim _{(\nu, \kappa) \rightarrow 0} \epsilon^{-1}\left(\left\|\mathbf{u}_{0}^{\nu, \kappa}-\mathbf{v}_{0}\right\|_{H^{s-2}}+\|\right.$ $\left.\theta_{0}^{v, \kappa}-\rho_{0} \|_{H^{s-2}}\right)=0$,

(3) $\lim _{(\nu, \kappa) \rightarrow 0} \epsilon^{-2}(\nu+\kappa)=0$.

Hence, combining the previous convergence results, it is easy to obtain from (75) that

$$
\lim _{(\nu, \mathcal{K}) \rightarrow 0}\left(\left\|\mathbf{w}^{\nu, \kappa}\right\|_{C\left([0, T] ; H^{s}\right)}+\left\|\chi^{\nu, \mathcal{K}}\right\|_{C\left([0, T] ; H^{s}\right)}\right)=0 .
$$

Step 6. By now, we have proved that (4), (5), and (7) hold on the time interval $[0, T]$. Now our aim is to show that these three results still hold on $\left[0, T_{0}\right]$. Define $T_{1}:=T$; now choose $\left(\mathbf{u}^{\nu, \mathcal{K}}\left(T_{1}\right), \theta^{\nu, \kappa}\left(T_{1}\right)\right)$ and $\left(\mathbf{v}\left(T_{1}\right), \rho\left(T_{1}\right)\right)$ as the new initial data, and one can see that the limit relation (3) still holds in the time $T_{1}$. Moreover, from Lemma 3, we know that $\left\|\mathbf{u}^{\nu, \mathcal{K}}\left(T_{1}\right)\right\|_{H^{s}}$, $\left\|\theta^{\nu, \mathcal{K}}\left(T_{1}\right)\right\|_{H^{s}},\left\|\mathbf{v}\left(T_{1}\right)\right\|_{H^{s}}$, and $\left\|\rho\left(T_{1}\right)\right\|_{H^{s}}$ depend only on the $H^{s}$ norm of the initial data $\left(\mathbf{v}_{0}, \rho_{0}\right)$. Then, we repeat the previous argument and find a positive sequence $\left\{T_{k}\right\}_{k=1}^{\infty}$ such that (4), (5), and (7) hold on $\left[0, T_{1}+\cdots+T_{k}\right]$. We assert that $\sum_{k=1}^{\infty} T_{k}=T^{*}$. Indeed, if $\sum_{k=1}^{\infty} T_{k}=\widetilde{T}<T^{*}$, and then the blow-up criterion implies that we can still extend $[0, \widetilde{T}]$ to some bigger interval, so we can continue this procedure as long as $\|\mathbf{v}(t)\|_{H^{s}}+\|\rho(t)\|_{H^{s}}<\infty$, and by the blow up criterion again, we get our assertion. Since $T_{0}<T^{*}$, after finite times iteration, we obtain the convergence results (4), (5), and (7).

Finally, since (7) holds, we have $\left\|\mathbf{u}^{\nu, \kappa}-\mathbf{v}\right\|_{C\left(\left[0, T_{0}\right] ; H^{s}\right)}+$ $\left\|\theta^{\nu, \kappa}-\rho\right\|_{C\left(\left[0, T_{0}\right] ; H^{s}\right)} \leq C$; then, the convergence result (6) follows from (5) and the following interpolation inequality:

$$
\|f\|_{H^{s^{\prime}}} \leq C\|f\|_{H^{s-1}}^{s-s^{\prime}}\|f\|_{H^{s}}^{1-\left(s-s^{\prime}\right)}, \quad s^{\prime} \in[s-1, s] .
$$

Therefore, we finish the proof of Theorem 1 .

\section{The $H^{s}$ Convergence Rate with Some Loss of Derivatives}

In this section, we will prove Theorem 2 , and we still use the same notations that are used in the proof of Theorem 1.

Proof of Theorem 2. Since (7) holds, without loss of generality, we may assume that

$$
\|\mathbf{u}\|_{C\left(\left[0, T_{0}\right] ; H^{s}\right)}+\|\theta\|_{C\left(\left[0, T_{0}\right] ; H^{s}\right)} \leq C
$$

for small $v$ and $\kappa$. By the extra regularity of the initial data $\left(\mathbf{v}_{0}, \rho_{0}\right)$, using the same reasonings that lead to (54) and the 
same extension method as Step 6 in the proof of Theorem 1, then we obtain

$$
\begin{gathered}
\left\|\mathbf{v}^{\epsilon}\right\|_{C\left(\left[0, T_{0}\right] ; H^{s+\delta}\right)}+\left\|\rho^{\epsilon}\right\|_{C\left(\left[0, T_{0}\right] ; H^{s+\delta}\right)} \leq C, \\
\left\|\mathbf{v}^{\epsilon}\right\|_{C\left(\left[0, T_{0}\right] ; H^{s+2}\right)}+\left\|\rho^{\epsilon}\right\|_{C\left(\left[0, T_{0}\right] ; H^{s+2}\right)} \leq C e^{C T_{0}} \epsilon^{\delta-2} .
\end{gathered}
$$

Now the proof is divided into two cases.

Case $1(0 \leq \delta \leq 2)$. In this case, the estimate (82) implies

$$
\left\|\mathbf{v}^{\epsilon}\right\|_{C\left(\left[0, T_{0}\right] ; H^{s+1}\right)}+\left\|\rho^{\epsilon}\right\|_{C\left(\left[0, T_{0}\right] ; H^{s+1}\right)} \leq C .
$$

Using the estimates $\left\|\widetilde{\mathbf{v}}^{\epsilon}\right\|_{L^{\infty}} \leq C\left\|\widetilde{\mathbf{v}}^{\epsilon}\right\|_{H^{s}}$ and (84), we deduce from the first inequality of (58) that

$$
\frac{1}{2} \frac{d}{d t}\left\|\widetilde{\mathbf{v}}^{\epsilon}\right\|_{H^{s}}^{2} \leq C\left\|\widetilde{\mathbf{v}}^{\epsilon}\right\|_{H^{s}}^{2}+\left\|\widetilde{\rho}^{\epsilon}\right\|_{H^{s}}\left\|\widetilde{\mathbf{v}}^{\epsilon}\right\|_{H^{s}}
$$

Similarly, one can infer from the first inequality of (59) that

$$
\frac{1}{2} \frac{d}{d t}\left\|\tilde{\rho}^{\epsilon}\right\|_{H^{s}}^{2} \leq C\left\|\tilde{\rho}^{\epsilon}\right\|_{H^{s}}^{2}+C\left\|\widetilde{\rho}^{\epsilon}\right\|_{H^{s}}\left\|\widetilde{\mathbf{v}}^{\epsilon}\right\|_{H^{s}} .
$$

The previous two inequalities together with (48) give

$$
\left\|\widetilde{\mathbf{v}}^{\epsilon}(t)\right\|_{H^{s}}+\left\|\widetilde{\rho}^{\epsilon}(t)\right\|_{H^{s}} \leq C e^{C T_{0}} \epsilon^{\delta}, \quad \forall t \in\left[0, T_{0}\right] .
$$

On the other hand, from (81) and the first inequality of (66), we can get

$$
\begin{aligned}
& \frac{1}{2} \frac{d}{d t}\left\|\mathbf{U}^{\epsilon}\right\|_{H^{s}}^{2} \leq C\left\|\mathbf{U}^{\epsilon}\right\|_{H^{s}}^{2} \\
& +\nu\left\|\mathbf{v}^{\epsilon}\right\|_{H^{s+2}}\left\|\mathbf{U}^{\epsilon}\right\|_{H^{s}}+\left\|\Theta^{\epsilon}\right\|_{H^{s}}\left\|\mathbf{U}^{\epsilon}\right\|_{H^{s}} \\
& \quad \leq C\left\|\mathbf{U}^{\epsilon}\right\|_{H^{s}}^{2}+C e^{C T_{0}} \nu \epsilon^{\delta-2}\left\|\mathbf{U}^{\epsilon}\right\|_{H^{s}} \\
& +\left\|\Theta^{\epsilon}\right\|_{H^{s}}\left\|\mathbf{U}^{\epsilon}\right\|_{H^{s}},
\end{aligned}
$$

where we have used (83) in the last step. Simultaneously, the $H^{s}$ energy estimate for $\Theta^{\epsilon}$ is estimated as

$$
\begin{gathered}
\frac{1}{2} \frac{d}{d t}\left\|\Theta^{\epsilon}\right\|_{H^{s}}^{2} \leq C\left\|\Theta^{\epsilon}\right\|_{H^{s}}^{2} \\
+C\left\|\Theta^{\epsilon}\right\|_{H^{s}}\left\|\mathbf{U}^{\epsilon}\right\|_{H^{s}} \\
+C e^{C T_{0}} \kappa \epsilon^{\delta-2}\left\|\Theta^{\epsilon}\right\|_{H^{s}}
\end{gathered}
$$

Then Gronwall inequality yields that

$$
\begin{aligned}
\left\|\mathbf{U}^{\epsilon}\right\|_{H^{s}}+\left\|\Theta^{\epsilon}\right\|_{H^{s}} & \\
\leq & e^{C T_{0} e^{C T_{0}}}\left(1+C T_{0} e^{C T_{0}}\right) \\
& \quad \times\left(\left\|\mathbf{U}^{\epsilon}(0)\right\|_{H^{s}}+\left\|\Theta^{\epsilon}(0)\right\|_{H^{s}}+(\kappa+\nu) \epsilon^{\delta-2}\right) .
\end{aligned}
$$

Combining (87) and (90), we can arrive at

$$
\begin{aligned}
& \|\mathbf{w}\|_{H^{s}}+\|\chi\|_{H^{s}} \\
& \quad \leq C_{4}\left(\|\mathbf{w}(0)\|_{H^{s}}+\|\chi(0)\|_{H^{s}}+(\kappa+\nu) \epsilon^{\delta-2}+\epsilon^{\delta}\right)
\end{aligned}
$$

for some $C_{4}>0$, from which we know that (8) holds by choosing $\epsilon^{\delta}=(\kappa+\nu) \epsilon^{\delta-2}$.

Case $2(0<\delta<1)$. In this case, we have

$$
\left\|v^{\epsilon}\right\|_{C\left(\left[0, T_{0}\right] ; H^{s+1}\right)}+\left\|\rho^{\epsilon}\right\|_{C\left(\left[0, T_{0}\right] ; H^{s+1}\right)} \leq C e^{C T_{0}} \epsilon^{\delta-1} .
$$

And also one obtains from (48) and the first inequality of (62)

$$
\begin{aligned}
& \left\|\widetilde{\mathbf{v}}^{\epsilon}\right\|_{L^{\infty}}+\left\|\widetilde{\rho}^{\epsilon}\right\|_{L^{\infty}} \\
& \quad \leq e^{C T_{0}}\left(\left\|\widetilde{v}^{\epsilon}(0)\right\|_{H^{s-2}}+\left\|\widetilde{\rho}^{\epsilon}(0)\right\|_{H^{s-2}}\right) \\
& \quad \leq C e^{C T_{0}} \epsilon^{2+\delta} .
\end{aligned}
$$

Applying the previous two estimates into (58) and (59), we get

$$
\begin{aligned}
& \frac{d}{d t}\left\|\widetilde{\mathbf{v}}^{\epsilon}\right\|_{H^{s}} \leq C\left\|\widetilde{\mathbf{v}}^{\epsilon}\right\|_{H^{s}}+C e^{C T_{0}} \epsilon^{1+2 \delta}+\left\|\widetilde{\rho}^{\epsilon}\right\|_{H^{s}}, \\
& \frac{d}{d t}\left\|\widetilde{\rho}^{\epsilon}\right\|_{H^{s}} \leq C\left\|\widetilde{\rho}^{\epsilon}\right\|_{H^{s}}+C e^{C T_{0}} \epsilon^{1+2 \delta},
\end{aligned}
$$

which yields that

$$
\begin{aligned}
\left\|\widetilde{\mathbf{v}}^{\epsilon}\right\|_{H^{s}}+\left\|\widetilde{\rho}^{\epsilon}\right\|_{H^{s}} & \leq e^{C T_{0} e^{C T_{0}}}\left(1+C T_{0} e^{C T_{0}}\right) \\
& \times\left(\left\|\widetilde{\mathbf{v}}^{\epsilon}(0)\right\|_{H^{s}}+\left\|\widetilde{\rho}^{\epsilon}(0)\right\|_{H^{s}}+\epsilon^{1+2 \delta}\right) \\
\leq & C e^{C T_{0} e^{C T_{0}}}\left(1+T_{0} e^{C T_{0}}\right) \epsilon^{\delta} .
\end{aligned}
$$

On the other hand, the estimates (71) and (93) imply

$$
\begin{aligned}
&\left\|\mathbf{U}^{\epsilon}\right\|_{L^{\infty}} \leq C^{\prime}\left(\phi_{1}(0)\right.\left.+\nu+\kappa+\epsilon^{2+\delta}\right), \\
& C^{\prime}:=\max \left\{C^{*}, C e^{C T_{0}}\right\} .
\end{aligned}
$$

Then, we insert this inequality and (83) and (92) into (66) and obtain

$$
\begin{aligned}
& \frac{d}{d t}\left\|\mathbf{U}^{\epsilon}\right\|_{H^{s}} \\
& \leq C\left\|\mathbf{U}^{\epsilon}\right\|_{H^{s}} \\
&+C C^{\prime} e^{C T_{0}} \epsilon^{\delta-1}\left(\phi_{1}(0)+\nu+\kappa+\epsilon^{2+\delta}\right) \\
&+C e^{C T_{0}} \nu \epsilon^{\delta-2}+\left\|\Theta^{\epsilon}\right\|_{H^{s}} .
\end{aligned}
$$

In the same way, the estimate (68) is replaced by

$$
\begin{aligned}
\frac{d}{d t}\left\|\Theta^{\epsilon}\right\|_{H^{s}} & \\
\leq & C\left\|\Theta^{\epsilon}\right\|_{H^{s}}+C\left\|\mathbf{U}^{\epsilon}\right\|_{H^{s}} \\
& +C C^{\prime} e^{C T_{0}} \epsilon^{\delta-1}\left(\phi_{1}(0)+\nu+\kappa+\epsilon^{2+\delta}\right) \\
& +C e^{C T_{0}} \kappa \epsilon^{\delta-2} .
\end{aligned}
$$


Hence, by the Gronwall inequality, we have

$$
\begin{aligned}
& \left\|\mathbf{U}^{\epsilon}\right\|_{H^{s}}+\left\|\Theta^{\epsilon}\right\|_{H^{s}} \\
& \leq C^{\prime \prime}\left(\left\|\mathbf{U}^{\epsilon}(0)\right\|_{H^{s}}+\left\|\Theta^{\epsilon}(0)\right\|_{H^{s}}\right. \\
& \left.\quad+\epsilon^{\delta-1} \phi_{1}(0)+(\nu+\kappa) \epsilon^{\delta-2}+\epsilon^{1+2 \delta}\right)
\end{aligned}
$$

for some $C^{\prime \prime}>0$. This inequality, together with (95), gives

$$
\begin{aligned}
\|\mathbf{w}\|_{H^{s}}+\|\chi\|_{H^{s}} & \\
\leq C_{5}\left(\|\mathbf{w}(0)\|_{H^{s}}+\|\chi(0)\|_{H^{s}}\right. & \\
& \left.\quad+\epsilon^{\delta-1} \phi_{1}(0)+(\nu+\kappa) \epsilon^{\delta-2}+\epsilon^{\delta}\right)
\end{aligned}
$$

for some $C_{5}>0$. So, (9) follows from (100) provided that we choose $\epsilon$ satisfying $\epsilon=\phi_{1}(0)+(\kappa+\nu)^{(1 / 2)}$.

\section{Acknowledgments}

The author would like to thank the referee's valuable suggestions which improved the presentation considerably. This work is supported by the National Science Foundation (Grant no. 11271334), Zhejiang Provincial Natural Science Foundation of China (Grant no. LY12A01019) and the Science Research Foundation of Jiaxing University.

\section{References}

[1] A. Majda, Introduction to PDEs andWaves for the Atmosphere and Ocean, vol. 9 of Courant Lecture Notes in Mathematics, AMS/CIMS, 2003.

[2] J. Pedlosky, Geophysical Fluid Dynamics, Springer, New York, NY, USA, 1987.

[3] J. R. Cannon and E. DiBenedetto, "The initial value problem for the Boussinesq equations with data in $L^{p}$," in Approximation Methods for Navier-Stokes Problems, vol. 771 of Lecture Notes in Mathematics, pp. 129-144, Springer, Berlin, Germany, 1980.

[4] D. Chae and H.-S. Nam, "Local existence and blow-up criterion for the Boussinesq equations," Proceedings of the Royal Society of Edinburgh $A$, vol. 127, no. 5, pp. 935-946, 1997.

[5] D. Chae, "Global regularity for the 2D Boussinesq equations with partial viscosity terms," Advances in Mathematics, vol. 203, no. 2, pp. 497-513, 2006.

[6] C. Foias, O. Manley, and R. Temam, "Attractors for the Bénard problem: existence and physical bounds on their fractal dimension," Nonlinear Analysis. Theory, Methods \& Applications, vol. 11, no. 8, pp. 939-967, 1987.

[7] B. Guo, "Spectral method for solving two-dimensional NewtonBoussinesq equations," Acta Mathematicae Applicatae Sinica, vol. 5, pp. 27-50, 1989.

[8] B. Guo, "Nonlinear Galerkin methods for solving twodimensional Newton-Boussinesq equations," Chinese Annals of Mathematics B, vol. 16, no. 3, pp. 379-390, 1995.

[9] T. Y. Hou and C. Li, "Global well-posedness of the viscous Boussinesq equations," Discrete and Continuous Dynamical Systems A, vol. 12, no. 1, pp. 1-12, 2005.

[10] Y. Taniuchi, "A note on the blow-up criterion for the inviscid 2-D Boussinesq equations," in The Navier-Stokes Equations:
Theory and Numerical Methods, vol. 223 of Lecture Notes in Pure and Applied Mathematics, pp. 131-140, Dekker, New York, NY, USA, 2002.

[11] P. Constantin, "Note on loss of regularity for solutions of the 3D incompressible Euler and related equations," Communications in Mathematical Physics, vol. 104, no. 2, pp. 311-326, 1986.

[12] P. Constantin, "On the Euler equations of incompressible fluids," American Mathematical Society, vol. 44, no. 4, pp. 603-621, 2007.

[13] A. Dutrifoy, "On 3-D vortex patches in bounded domains," Communications in Partial Differential Equations, vol. 28, no. 7-8, pp. 1237-1263, 2003.

[14] T. Kato, "Nonstationary flows of viscous and ideal fluids in $\mathbb{R}^{3}$," vol. 9, pp. 296-305, 1972.

[15] N. Masmoudi, "Remarks about the inviscid limit of the NavierStokes system," Communications in Mathematical Physics, vol. 270, no. 3, pp. 777-788, 2007.

[16] N. Masmoudi, "Examples of singular limits in hydrodynamics," in Handbook of Differential Equations, pp. 195-275, NorthHolland, Amsterdam, The Netherlands, 2007.

[17] H. S. G. Swann, "The convergence with vanishing viscosity of nonstationary Navier-Stokes flow to ideal flow in $R^{3}$," Transactions of the American Mathematical Society, vol. 157, pp. 373-397, 1971.

[18] R. R. Coifman and Y. Meyer, "Nonlinear harmonic analysis, operator theory and P.D.E," in Beijing Lectures in Harmonic Analysis, vol. 112, pp. 3-45, Princeton University Press, Princeton, NJ, USA, 1986.

[19] T. Kato, "Liapunov functions and monotonicity in the NavierStokes equation," in Functional-Analytic Methods for Partial Differential Equations, vol. 1450, pp. 53-63, Springer, Berlin, Germany, 1990.

[20] A. J. Majda and A. L. Bertozzi, Vorticity and Incompressible Flow, vol. 27, Cambridge, UK, 2002. 


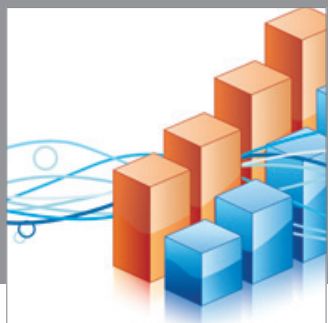

Advances in

Operations Research

mansans

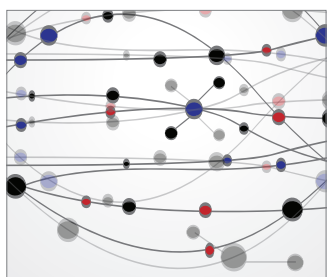

The Scientific World Journal
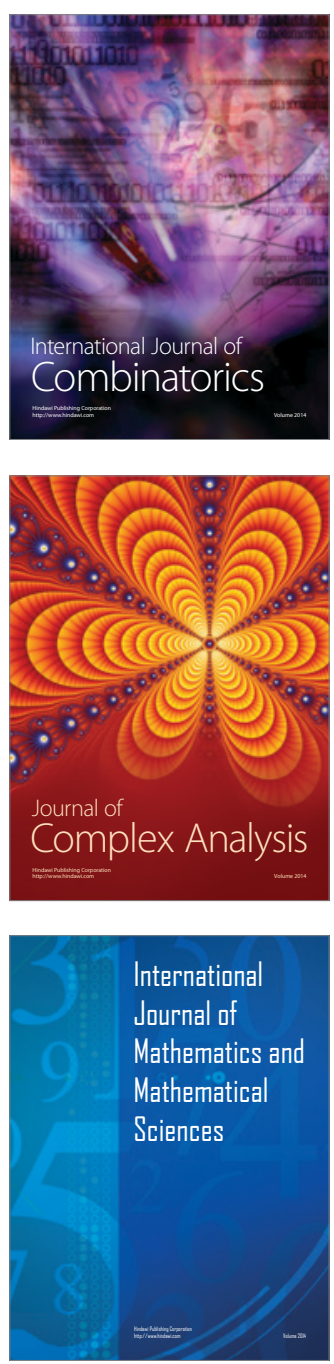
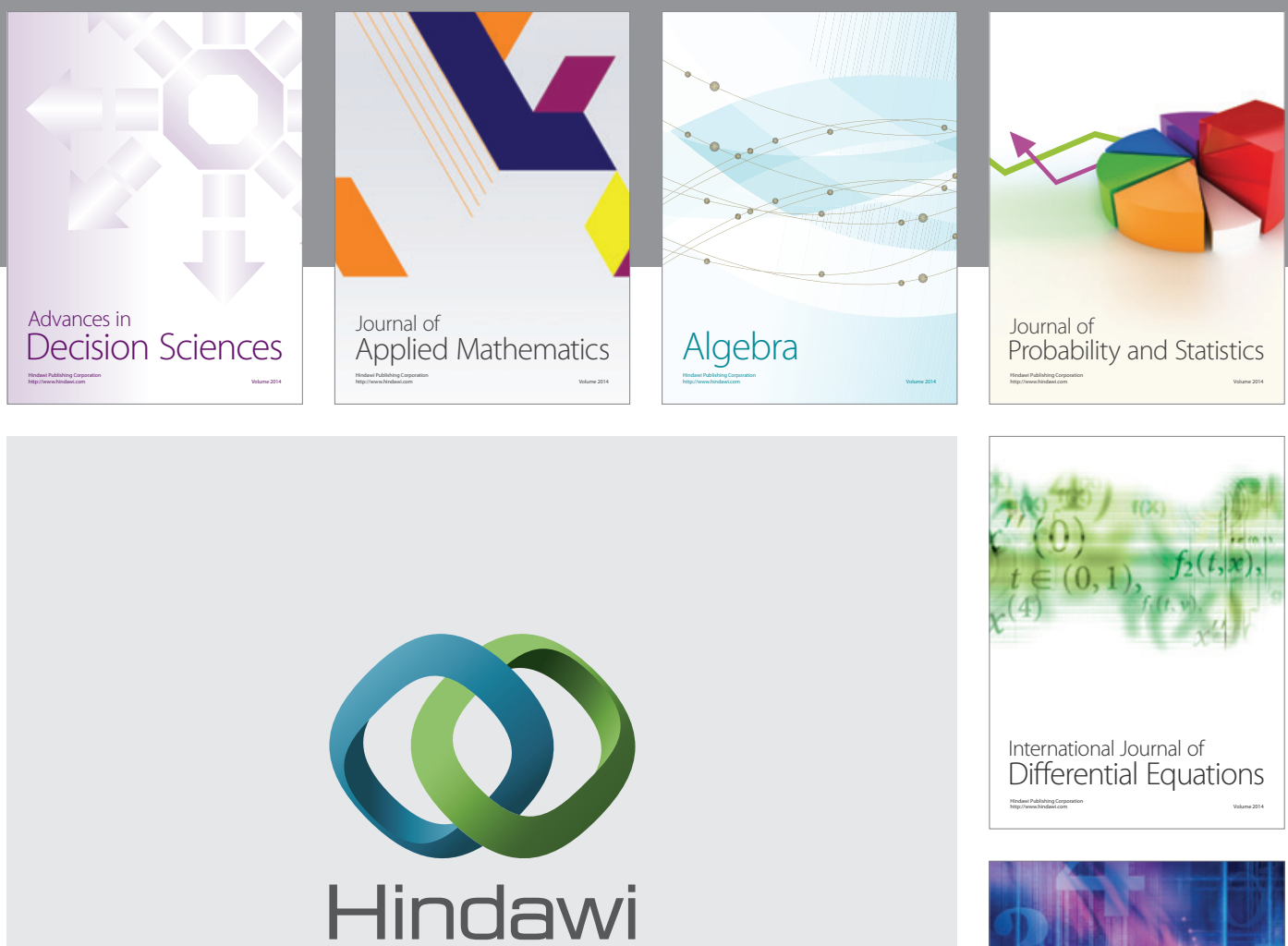

Submit your manuscripts at http://www.hindawi.com
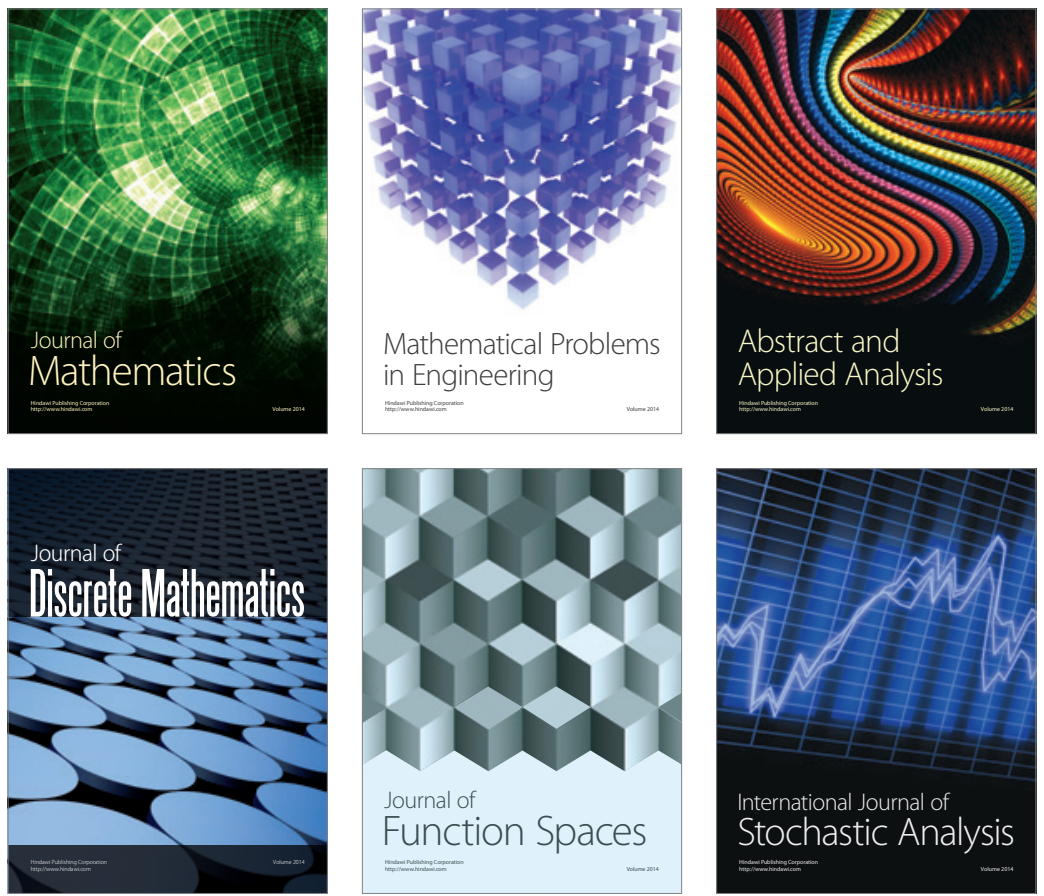

Journal of

Function Spaces

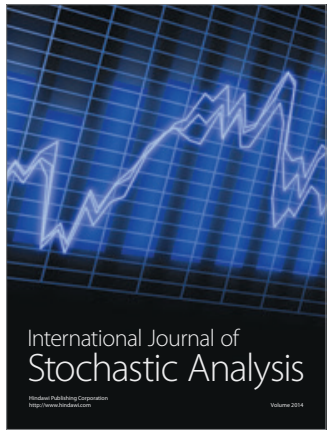

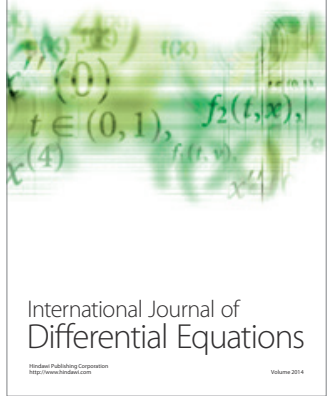
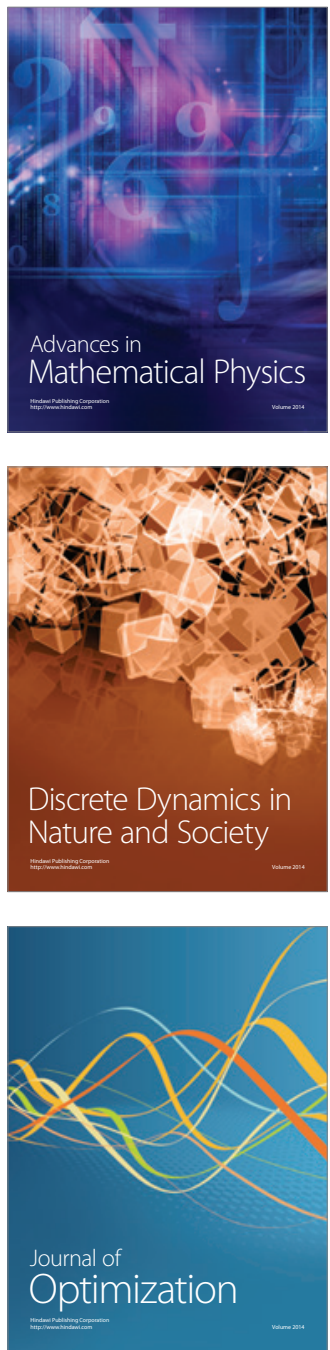